Recepción: 20 / 04 / 2017

Aceptación: 20 / 05 / 2017

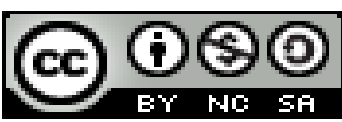

Ciencias de la Educación

Publicación: 15 / 06 / 2017

Artículo de Investigación

\title{
El desarrollo de destrezas y habilidades en la motricidad gruesa en niños y niñas de 24 a 36 meses
}
The development of skills and abilities in gross motor skills in children between 24 and 36 months

\section{O desenvolvimento de competências e habilidades em habilidades motoras em crianças de 24 a 36 meses}

\author{
María F. Constante-Barragan ${ }^{\mathrm{I}}$ \\ maria.constante@utc.edu.ec \\ Adriana G. Rengifo-Tapia II \\ adriana.rengifo@hotmail.com \\ Leslie A. Herrera- Yugcha III \\ leslie.herrera@hotmail.com
}

Correspondencia: maria.constante@utc.edu.ec

Magister en Ciencias de la Educación Mención Educación Parvulario; Magister en Ciencias de la Educación Mención en Gestión Educativa y Desarrollo Social; Profesora de Educación Parvulario; Licenciada en Ciencias de la Educación Mención educación Parvularia; Universidad de Cotopaxi, Cotopaxi, Ecuador.

II. Licenciada en Ciencias de la educación Mención educación Parvularia, Universidad de Cotopaxi, Cotopaxi, Ecuador.

III. Licenciada en Ciencias de la educación Mención educación Parvularia, Universidad de Cotopaxi, Cotopaxi, Ecuador

http://polodelconocimiento.com/ojs/index.php/es/article/view/154 


\section{Resumen}

La presente investigación tiene como finalidad comprender y resaltar la importancia que ejerce el desarrollo psicomotor en los niños y niñas y su influencia que tiene en la etapa escolar una adecuada estimulación en el áreas motriz gruesa, es por eso que se ha considerado este punto de vista, debido a que el niño está en un proceso de aprendizaje mediante sus sentidos a través del movimiento, para adquirir un nuevo aprendizaje. Buscando así determinar la importancia de la estimulación motriz mediante la adquisición de habilidades y destrezas motoras gruesas para desarrollar un adecuado proceso en los elementos que constituyen la psicomotricidad, basándose en ciertas problemáticas que existe en la actualidad donde la motricidad gruesa no tiene un área determinada para trabajar con los niños sin presentar una planificación especifica en esta área por lo que se observó el deficiente desarrollo que presentan los niños en esta área. La entrevista, encuesta y la ficha de observación fueron los instrumentos que permitieron recopilar información para medir y aportar sobre la importancia del desarrollo psicomotor en los niños, los materiales que se utilizó para aplicar la ficha de observación fueron los adecuados para la edad de los niños permitiéndonos evaluar el nivel de su desarrollo psicomotriz. La investigación fue de carácter descriptiva-exploratoria porque de esta manera se pudo identificar los niveles de desarrollo de los niños en el Centro Infantil del Buen Vivir Infancia Feliz, la teoría de Piaget afirma que la inteligencia se construye a partir de la actividad motriz, es aquí donde relacionamos la importancia de la psicomotricidad durante los primeros años de vida de los niños, esta etapa es la que se formaran gran parte de los aprendizajes de los niños y servirá como base a los que se irán presentando a largo de su vida.

Palabras claves: Motricidad gruesa, desarrollo, psicomotor, habilidades, destrezas. 


\begin{abstract}
The present research aims to understand and highlight the importance of psychomotor development in children and their influence in the school stage an adequate stimulation in the gross motor areas, which is why this point of view has been considered, Because the child is in a process of learning through his senses through movement, to acquire a new learning. In this way, it is possible to determine the importance of motor stimulation through the acquisition of gross motor skills and abilities to develop an adequate process in the elements that constitute the psychomotricity, based on certain problems that exist today where gross motor skills do not have a specific area for Work with the children without presenting a specific planning in this area so that was observed the deficient development that the children present in this area. The interview, survey and observation sheet were the instruments that allowed to gather information to measure and contribute on the importance of psychomotor development in children, the materials that were used to apply the observation sheet were appropriate for the age of the children Allowing us to evaluate the level of their psychomotor development. The research was descriptive-exploratory in nature because in this way it was possible to identify the levels of development of children in the Children's Center of Good Living Happy Childhood, Piaget's theory states that intelligence is built from the motor activity, is Here we relate the importance of psychomotricity during the first years of life of children, this stage is what will form much of the learning of children and will serve as a base to those who will be presented throughout his life.
\end{abstract}

Key words: Gross motor, development, psychomotor, skills, skills. 


\section{Resumo}

Esta pesquisa tem como objetivo compreender e destacar a importância do desenvolvimento de exercícios psicomotor em crianças e influenciar tem no palco escola estimulação adequada nas áreas motoras, razão pela qual tem sido considerado este ponto de vista porque a criança está em um processo de aprendizagem através de seus sentidos através do movimento, para adquirir um novo aprendizado. e procurando determinar a importância da estimulação motora através da aquisição de competências e habilidades motoras a desenvolver um processo adequado os elementos que constituem psicomotor com base em determinados problemas que existem hoje, onde motora grossa não tem uma área para trabalhar com crianças sem apresentar planejamento específico nesta área para que as crianças pobres têm foi observado desenvolvimento nesta área. Entrevista, pesquisa e folha de observação foram os instrumentos que permitiram a coleta de informações para medir e trazer sobre a importância do desenvolvimento psicomotor em crianças, os materiais utilizados para implementar a folha de observação foram adequados para a idade das crianças o que nos permite avaliar o nível de desenvolvimento psicomotor. A pesquisa foi de caráter descritivo-exploratório, pois desta forma, foi possível identificar os níveis de desenvolvimento das crianças no Centro de Bem Viver feliz Infância Infantil, a teoria de Piaget diz que a inteligência é construída a partir da atividade motora é onde relacionar a importância de habilidades psicomotoras, durante os primeiros anos de vida das crianças, nesta fase, é que grande parte da aprendizagem de crianças foram formadas e servir como base no qual será introduzido em sua vida.

Palavras-chave: Desenvolvimento motor grosseiro, psicomotor, habilidades. 


\section{Introducción.}

La presente investigación da importancia a los primeros años de vida en el desarrollo del ser humano a fin de dar una atención adecuada a los niños/as tomando el área motriz gruesa, las necesidades y dificultades que esta presenta en los infantes siendo estos una inadecuada estimulación lo que no logra desarrollar en su totalidad las destrezas y habilidades motoras gruesas dificultando en el desarrollo integral del niño. (Cerdas Núñez, Polanco Hernández, \& Rojas Núñez, 2002) (Lev Vygotsky, 1985)

El mismo que está dirigido para niños/as de 24 a 36 meses de edad, en donde se propone a futuro adecuar el área de motricidad gruesa y la creación de un manual de ejercicios dirigidos al área motriz, en el sitio donde se realiza la investigación en el Barrio Patután en el CIBV Infancia Feliz, se ha detectado que existen niños/as que necesitan obtener atención integral combinando aspectos de salud, nutrición, desarrollo motor, afectivo; lo cual es deficiente por la falta de conocimiento en la comunidad y dentro del núcleo familiar, dichos niños/as se encuentran al cuidado de madres comunitarias las mismas que no tienen conocimiento de cómo cuidar y estimular para desarrollar una motricidad gruesa adecuada. (Osorio, Torres-Sánchez, Hernández, López-Carrillo, \& Schnaas, 2010) (Black, Baqui, Zaman, McNary, \& Arifeen, 2007)

En el mencionado sector en la actualidad existen espacios verdes reducidos no acorde para el desarrollo de los niños/as además podemos notar que no existe personal capacitado por la falta de interés de estas personas para capacitarse por ello cuentan con una escasa metodología para la enseñanza aprendizaje; la constante despreocupación familiar que no dan prioridad a la formación de sus hijos por tener un bajo nivel de instrucción y es por eso que se ve un desconocimiento de lo importante que es el correcto desarrollo de la motricidad gruesa en los infantes y a su vez la falta de 
tiempo por parte de los mismos para compartir con sus hijos, ha venido creando problemas como, falta de integración grupal, tonicidad muscular, tímidos poco expresivos. (Pic, 1989) (Schilder, 1982)

Por lo tanto parte de aquí la curiosidad y la motivación de investigar el problema y dar intentos de solución para desarrollar en los niños las capacidades motoras, afectivas, social, cognitivo las mismas que permitirán un verdadero desarrollo psicomotriz.

También se incorporara información actualizada sobre el tema para fundamentar científicamente esta posición, en momentos en que resulte complicada la aplicación.

Es fundamental e indispensable que la Guardería del Barrio Patután cuente con un manual de ejercicios psicomotrices que sirva de guía didáctica para lograr con esto, y una adecuación del rincón de motricidad gruesa alcanzar el éxito en el desenvolvimiento de los niños/as.

En la investigación el objeto de estudio es el determinar la importancia de la estimulación motriz para mejorar la adquisición de habilidades y destrezas motoras gruesas de acuerdo a la edad de los niños y niñas de 24 a 36 meses en el CIBV Infancia Feliz.

Las técnicas de investigación que ayudaron a recopilar datos durante la investigación para nuestra propuesta son: entrevistas, encuesta y ficha de observación.

Utilizamos la metodología mixta ya que el enfoque de la investigación es cualicuantitativo empleando procesos cuidadosos, sistemáticos y prácticos para generar conocimiento, se va analizar la motricidad gruesa tomando en énfasis el estudio de las habilidades y destrezas integras desde el punto de vista cualitativo, se basara en los resultados de la observación de los niños y niñas de 24 a 36 meses del CIBV Infancia Feliz siendo este la parte cuantitativa 


\section{Materiales y métodos.}

\section{Mixta: Cualicuantitativa}

El enfoque de la investigación es cualitativo y cuantitativo porque se emplean procesos cuidadosos, sistemáticos y prácticos para generar conocimiento, se va analizar la Motricidad gruesa tomando en énfasis el estudio central en la lateralidad, equilibrio, coordinación y rapidez desde el punto de vista cualitativo, se basara en los resultados de la observación de los niños y niñas en el CIBV Infancia Feliz.

SABINO, C. (1992) precisa a la investigación cualitativa como: "El punto de referencia, directo o indirecto, imprescindible para la comprensión profunda de los problemas. Los diseños cualitativos, exclusivos de este campo del conocimiento, intentan recuperar para el análisis parte de esta complejidad del sujeto y de sus modos de ser y de hacer en el medio que lo rodea. Lo íntimo, lo subjetivo, por definición difícilmente cuantificables, son el terreno donde se mueven por lo tanto son métodos cualitativos”. (pág. 73)

El contacto directo con los actores y con los escenarios en los cuales tiene lugar la producción de significados, culturales, sociales y personales lo que permite descubrir o reconocer los conflictos y las fracturas, las divergencias y los consensos, las regularidades e irregularidades, las diferencias y homogeneidades de la estructura social edificada en un contexto concreto; así como realización personal, social y cultural en dicho contexto, todo esto concebido desde la perspectiva de investigación cualitativa". (p. 43)

Modalidad Básica de Investigación.

La investigación tiene las siguientes modalidades: Bibliográfica

\section{Resultados.}

Análisis e interpretación de la entrevista realizadas a la autoridad (coordinadora) del CIBV

\section{Infancia Feliz.}


La coordinadora Victoria Valladares y la docente Cecilia Yanchatipan a cargo de los niños de 24 a 36 meses nos manifiestan que los aspectos con los que trabajan en la motricidad gruesa es a base de los recursos con los que cuenta el centro infantil estos pueden ser evaluaciones que se realizan a los niños ates del ingreso al CIVB, los juegos que se realizan para el área motriz gruesa son juegos tradicionales saltar la cuerda, el baile de la silla y la rayuela. Los componentes que deben tener para estimular la motricidad gruesa seria la interacción de todos los niños, que sea un juego participativo; las actividades lúdicas que se pueden proponer para un desarrollo adecuado de la locomoción en el infante serian juegos de lanzar y atrapar, juegos de obstáculos, marchar coordinando pies y manos, juegos equilibrio y ritmo ya que estos ayudaran a que el niño consiga una mejor coordinación en sus movimientos.

También nos dan a conocer que los medios físicos y los recursos que ofrece el CIBV no están adecuados para realizar ejercicios de motricidad.

Análisis e interpretación de la encuesta aplicada a los Padres de Familia del CIBV "Infancia Feliz”.

\begin{tabular}{|l|l|l|}
\hline INDICADOR & FRECUENCIA & PORCENTAJE \\
\hline SI & 2 & $12 \%$ \\
\hline NO & 23 & $88 \%$ \\
\hline TOTAL & $\mathbf{2 5}$ & $\mathbf{1 0 0 \%}$ \\
\hline
\end{tabular}

\section{1.- Ud. Ha realizado estimulación a su niño antes del ingreso al CIVB \\ TABLA N.-1 ESTIMULACION FUENTE: Padres de Familia del CIVB Infancia Feliz}




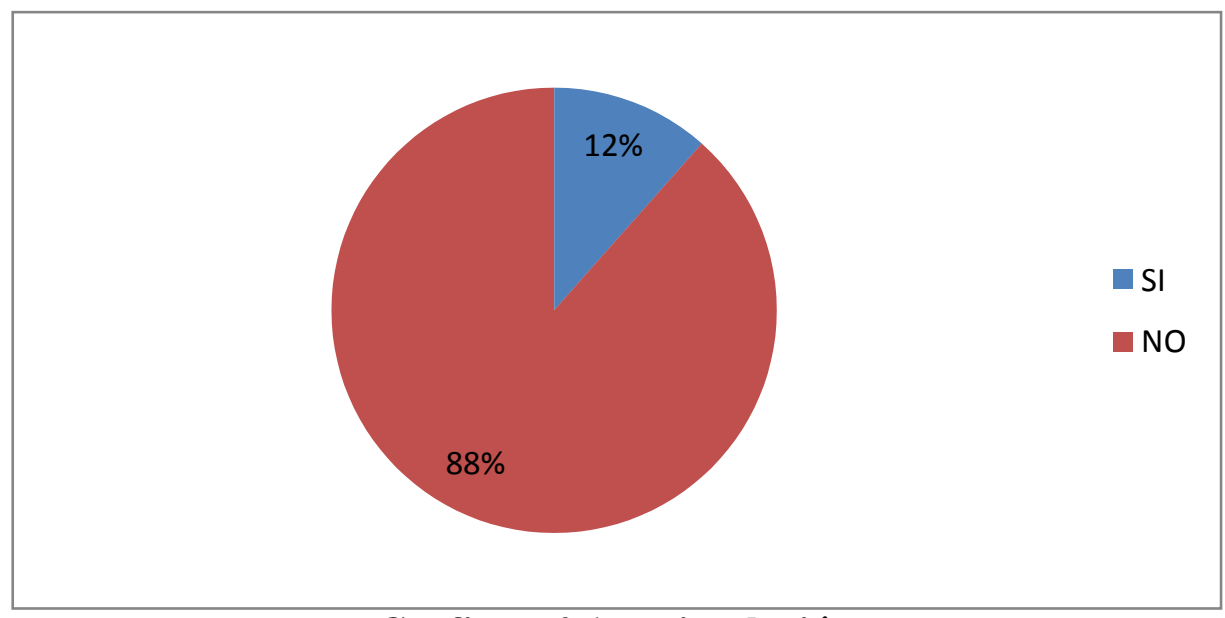

Grafico n $^{\circ}$ 1: estimulación

Fuente: padres de familia del CIVB infancia feliz

\section{ANÁLISIS}

De la encuesta realizada a los padres de familia en el CIVB Infancia Feliz el $12 \%$ han realizado estimulación antes del ingreso al CIVB y el $85 \%$ no han realizado ningún tipo de estimulación a su niño.

Es importante que exista una adecuada estimulación en el área motriz ya que, es vital para el desarrollo integral del niño, incluyendo los movimientos de brazos, piernas, espalda, abdomen; permitiendo que el niño se mueva y se desplace para explorar y conocer el mundo que le rodea.

\begin{tabular}{|l|l|l|}
\hline INDICADOR & FRECUENCIA & PORCENTAJE \\
\hline SI & 15 & $60 \%$ \\
\hline NO & 10 & $40 \%$ \\
\hline TOTAL & $\mathbf{2 5}$ & $\mathbf{1 0 0 \%}$ \\
\hline
\end{tabular}

2.-Sabe si su niño desarrolla adecuadamente la motricidad gruesa

TABLA N.-2 DESARROLLO (MOTRICIDAD GRUESA)

FUENTE: Padres de Familia del CIVB Infancia Feliz 


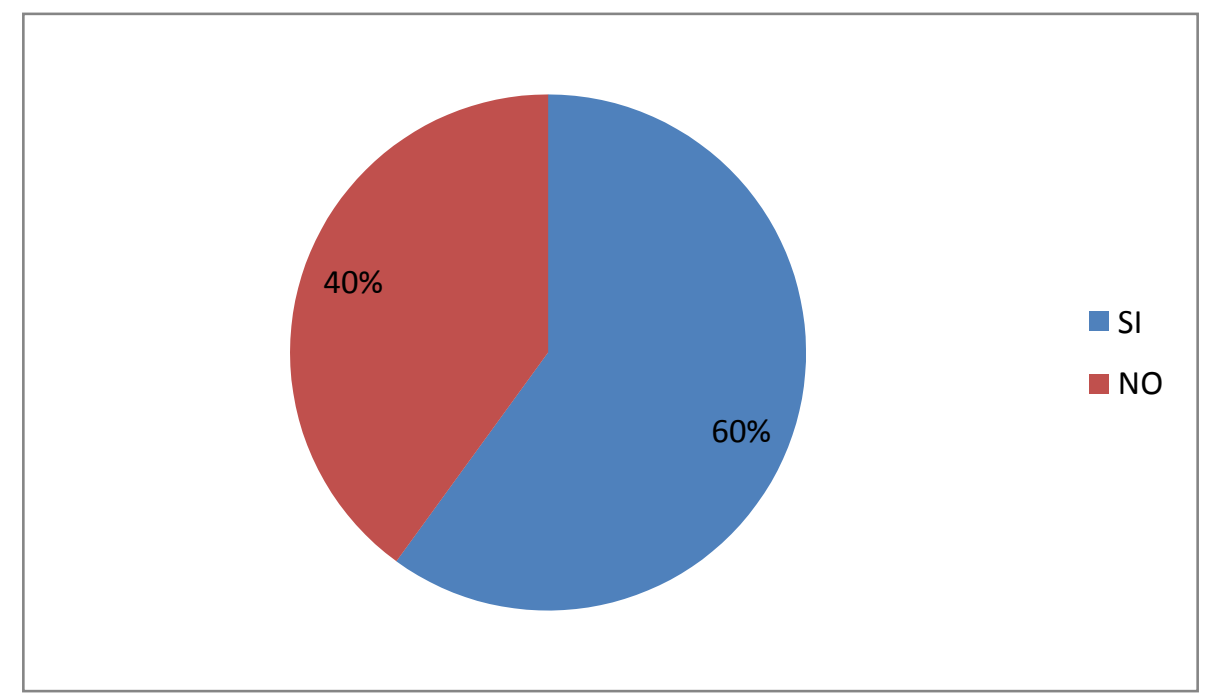

Grafico $n^{\circ}$ 2: desarrollo (motricidad gruesa)

Fuente: padres de familia del CIVB infancia feliz

\section{ANÁLISIS}

El $60 \%$ de los padres de familia de los niños/as del mismo CIVB consideran que ha desarrollado una adecuada motricidad gruesa mientras que el $40 \%$ consideran que su niño/a no ha desarrollado una adecuada motricidad gruesa.

El desarrollo de la motricidad gruesa es de vital importancia en el desarrollo integral del niño; este tipo de motricidad compromete grandes grupos musculares y grandes segmentos del niño o incluso comprometer todo el cuerpo.

\begin{tabular}{|c|c|c|}
\hline INDICADOR & FRECUENCIA & PORCENTAJE \\
\hline SI & 12 & $48 \%$ \\
\hline NO & 13 & $52 \%$ \\
\hline TOTAL & $\mathbf{2 5}$ & $\mathbf{1 0 0 \%}$ \\
\hline
\end{tabular}

3.-Ha realizado algún test donde indica los avances de motricidad gruesa dependiendo la edad del niño

TABLA N.-3 TEST DE AVANCES (MOTRICIDAD GRUESA)

FUENTE: Padres de Familia del CIVB Infancia Feliz 


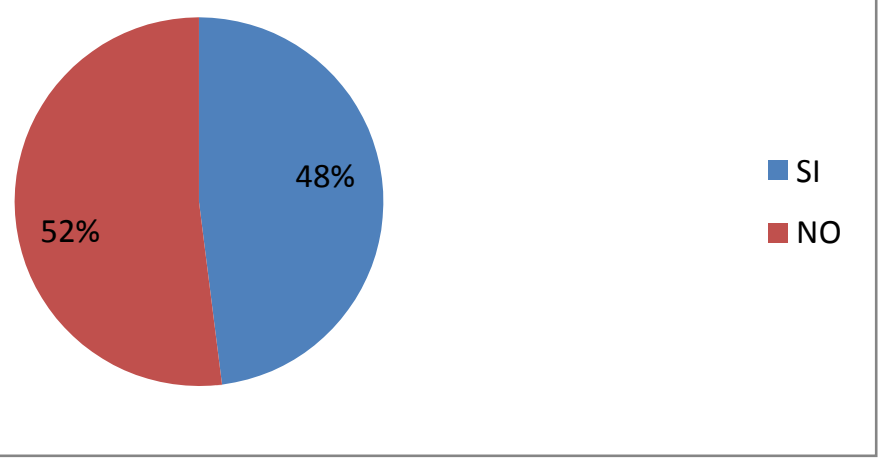

Grafico ${ }^{\circ}$ 3: test de avances (motricidad gruesa) Fuente: padres de familia del CIVB infancia feliz

\section{ANÁLISIS}

El $48 \%$ de los padres de familia del CIVB mencionan que han realizado un test que indica los avances que tiene su niño en motricidad gruesa, mientras que el 52\% mencionan que no han realizado ningún tipo de test en motricidad gruesa.

Es considerado de importancia el realizar un test de motricidad gruesa ya que este nos ayuda analizar el nivel del niño en su parte motriz gruesa ya que, comprende todo lo relacionado con el desarrollo cronológico del niño especialmente en el crecimiento del cuerpo y de las habilidades psicomotrices, es decir se refiere a todos aquellos movimientos de la locomoción o del desarrollo postural como andar, correr, saltar.

\begin{tabular}{|c|c|c|}
\hline INDICADOR & FRECUENCIA & PORCENTAJE \\
\hline SI & 5 & $20 \%$ \\
\hline NO & 20 & $80 \%$ \\
\hline TOTAL & 25 & $100 \%$ \\
\hline
\end{tabular}

4.-Considera Ud. Que los recursos con los que cuenta el CIVB son adecuados para un desarrollo integro de su niño

TABLA N.-4 RECURSOS

FUENTE: Padres de Familia del CIVB Infancia Feliz 


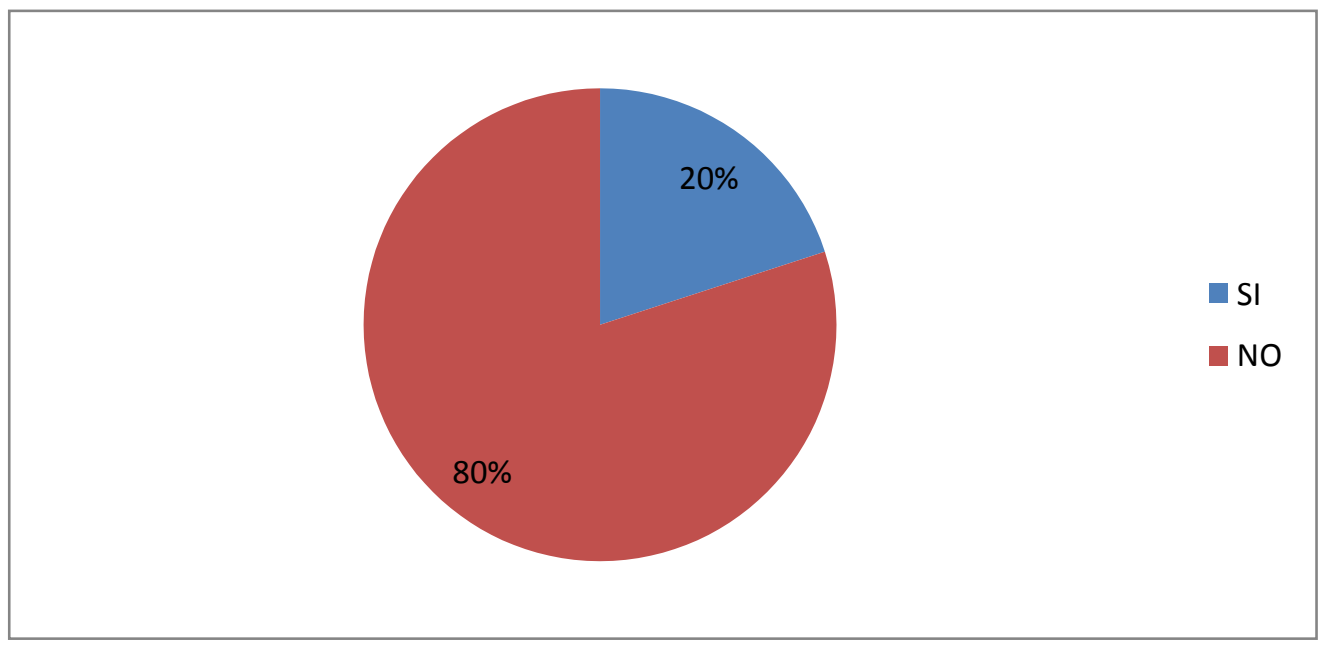

\section{Grafico n ${ }^{\circ}$ 4: recursos}

Fuente: padres de familia del CIVB infancia feliz

\section{ANÁLISIS}

El 20\% de los padres de familia consideran que le CIVB cuenta con los recursos adecuados para el desarrollo integral de su niño/a, mientras que el $80 \%$ de los padres de familia afirma que el CIVB no cuenta con los recursos para el desarrollo adecuado de su niño.

Es importante que para que los niños/as tenga un óptimo desarrollo en el área motriz gruesa el CIVB debe contar con los recursos necesarios, para de esta manera poder desarrollar al máximo todas las habilidades y destrezas motoras gruesa.

\begin{tabular}{|l|l|l|}
\hline INDICADOR & FRECUENCIA & PORCENTAJE \\
\hline SI & 25 & $0 \%$ \\
\hline NO & 0 & $100 \%$ \\
\hline TOTAL & $\mathbf{2 5}$ & $\mathbf{1 0 0 \%}$ \\
\hline
\end{tabular}

5.- Ha visto avances en su niño en la motricidad gruesa

TABLA N.-5 AVANCES (MOTRICIDAD GRUESA) FUENTE: Padres de Familia del CIVB Infancia Feliz 


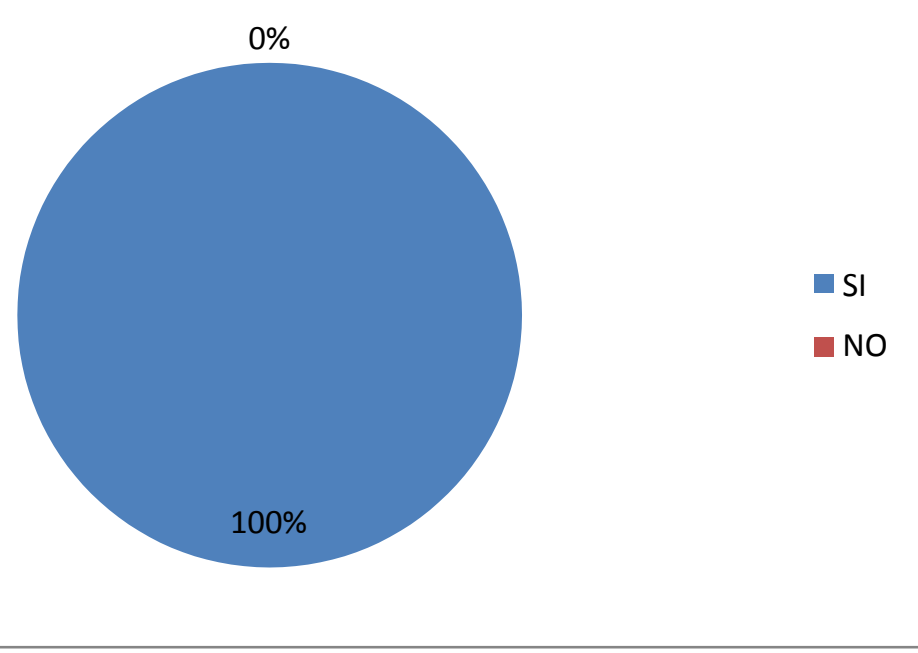

Grafico ${ }^{\circ}$ 5: avances (motricidad gruesa)

Fuente: padres de familia del CIVB infancia feliz

\section{ANÁLISIS}

El $100 \%$ de los padres de familia mencionan que han visto notables avances en el desarrollo de la motricidad gruesa en su niño/a.

La Motricidad gruesa tiene que ver con los cambios de posición del cuerpo y la capacidad de mantener el equilibrio es de mucha importancia que exista una adecuada estimulación para obtener buenos resultados en el área motriz gruesa.

\begin{tabular}{|l|l|l|}
\hline \multirow{2}{*}{ INDICADOR } & FRECUENCIA & PORCENTAJE \\
\hline SI & 25 & \\
\hline NO & 0 & $100 \%$ \\
\hline TOTAL & 25 & $0 \%$ \\
\hline
\end{tabular}

6.-Desearia que su niño reciba terapias de estimulación infantil. TABLA N.-6 TERAPIAS DE ESTIMULACION TEMPARANA FUENTE: Padres de Familia del CIVB Infancia Feliz 


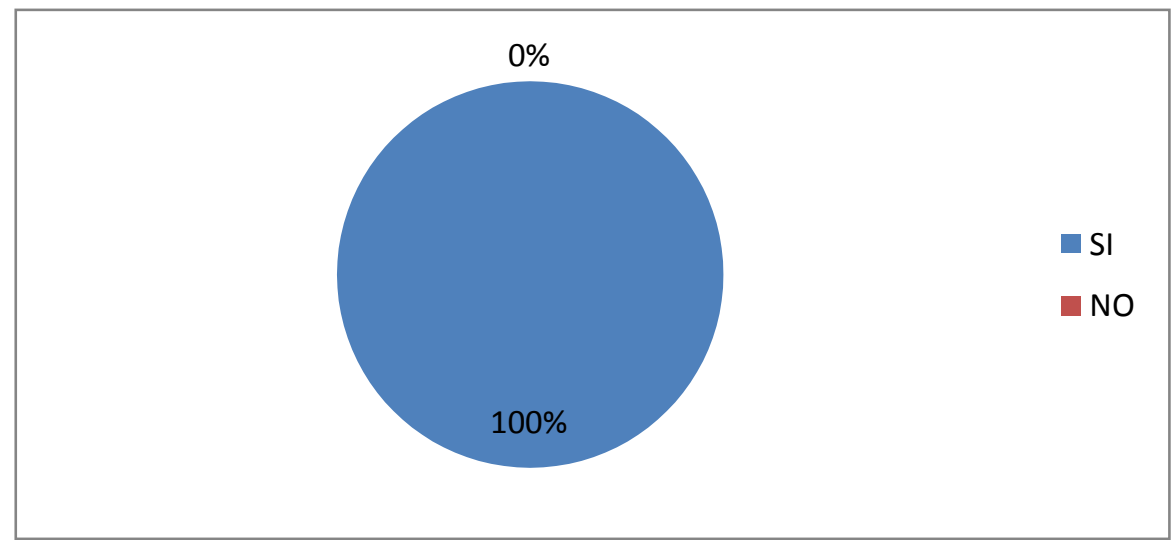

Grafico n ${ }^{\circ}$ 6: terapias de estimulación temprana

Fuente: padres de familia del CIVB infancia feliz

\section{ANÁLISIS}

El 100\% de los padres de familia del CIVB Infancia Feliz desearían que sus niños/as reciban estimulación infantil.

La importancia de la estimulación infantil es tal que se considera un requisito básico para el óptimo desarrollo del cerebro del niño, ya que potencia sus funciones cerebrales en todos los aspectos (cognitivo, lingüístico, motor y social)

\begin{tabular}{|l|l|l|}
\hline INDICADOR & FRECUENCIA & PORCENTAJE \\
\hline SI & 25 & $100 \%$ \\
\hline NO & 0 & $0 \%$ \\
\hline TOTAL & 25 & $100 \%$ \\
\hline
\end{tabular}

7.-Es importante trabajar en su niño la motricidad gruesa.

TABLA N.-7 MOTRICIDA GRUESA

FUENTE: Padres de Familia del CIVB Infancia Feliz 


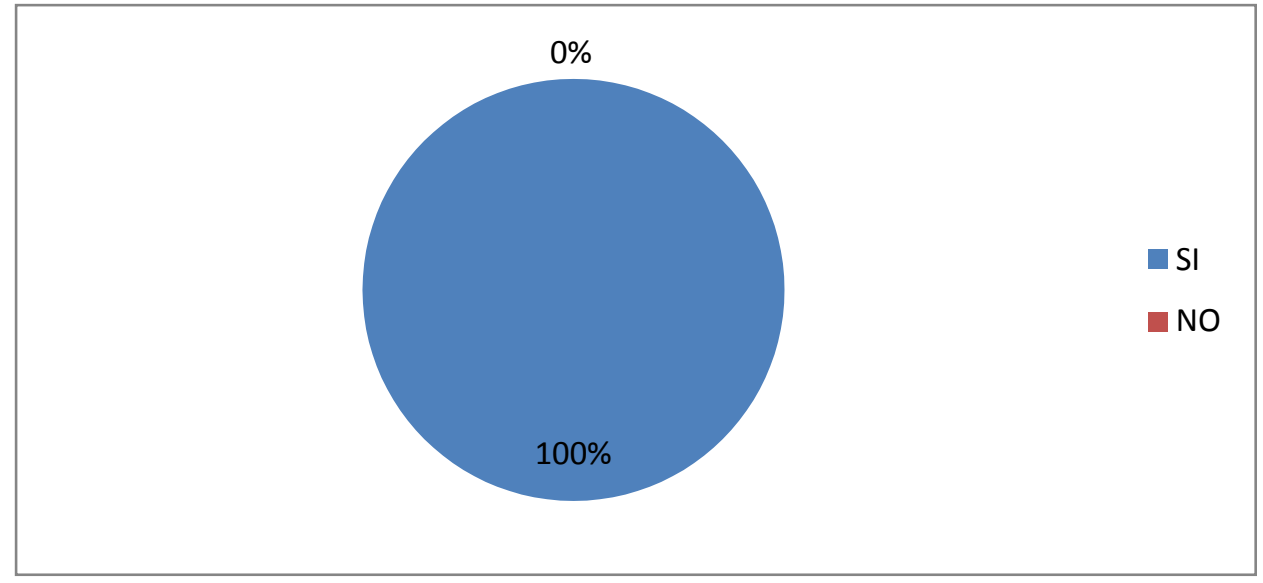

Grafico ${ }^{\circ}$ 7: motricidad gruesa

Fuente: padres de familia del CIVB infancia feliz

\section{ANÁLISIS}

El $100 \%$ de los padres de familia mencionan que es importante trabajar en su niño la motricidad gruesa.

Un desarrollo motriz apropiado para la edad del niño es especialmente importante en el ámbito de la motricidad gruesa, puesto que el estado de desarrollo afecta directamente otros ámbitos de la vida del niño. Un niño que desarrolla con normalidad su motricidad gruesa tendrá con seguridad menos problemas en la escuela para aprender a escribir y en las clases de gimnasia, con temas como el equilibrio. Podrá concentrarse mejor, tendrá más confianza en sí mismo y podrá reaccionar más deprisa. Estas habilidades le beneficiarán en su día a día.

\begin{tabular}{|l|l|l|}
\hline INDICADOR & FRECUENCIA & PORCENTAJE \\
\hline SI & 25 & $100 \%$ \\
\hline NO & 0 & $0 \%$ \\
\hline TOTAL & 25 & $100 \%$ \\
\hline
\end{tabular}

8.-Los diferentes ejercicios de motricidad gruesa ayudan a controlar y coordinar movimientos en su niño.

TABLA N.-8 EJERCICIOS DE MOTRICIDAD GRUESA FUENTE: Padres de Familia del CIVB Infancia Feliz 


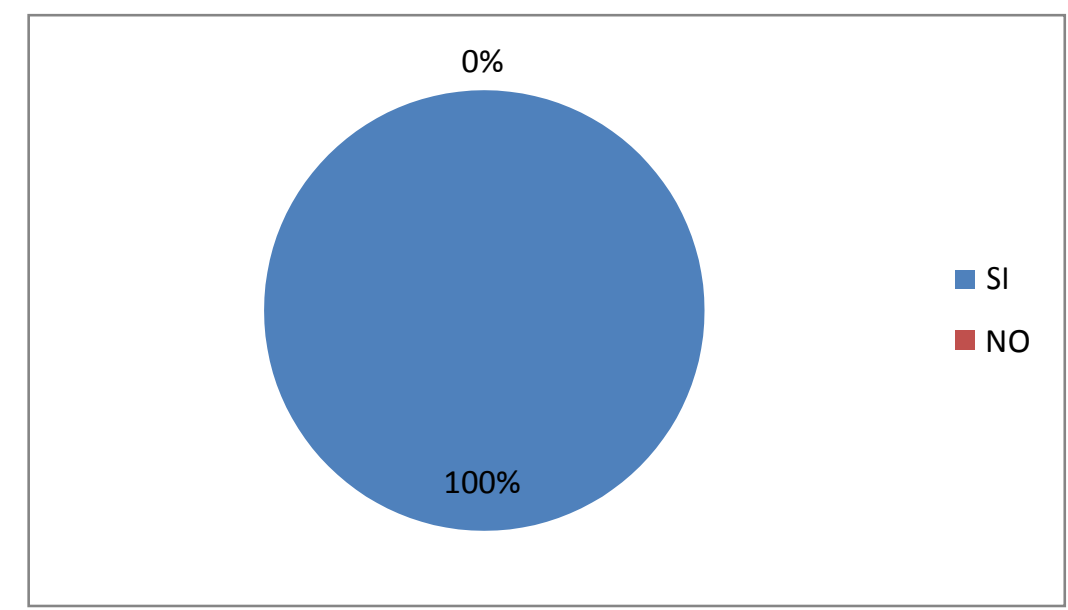

\section{GRAFICO $\mathrm{N}^{\circ}$ 8: EJERCICIOS DE MOTRICIDAD GRUESA FUENTE: Padres de Familia del CIVB Infancia Feliz}

\section{ANÁLISIS}

El $100 \%$ de los padres de familia mencionan que los diferentes ejercicios en motricidad gruesa ayudan a controlar y coordinar los movimientos en sus niños.

Al realizar ejercicios de motricidad gruesa adecuados a la edad del niño estamos ayudando a desarrollar un sentido de pertenencia e independencia ya que tienen además mejor dominio de sus cuerpos y movimientos.

\begin{tabular}{|l|l|l|}
\hline INDICADOR & FRECUENCIA & PORCENTAJE \\
\hline SI & 25 & $100 \%$ \\
\hline NO & 0 & $0 \%$ \\
\hline TOTAL & 25 & $100 \%$ \\
\hline
\end{tabular}

9.-La postura que tiene su niño para realizar actividades cotidianas son correctas TABLA N.-9 LA POSTURA FUENTE: Padres de Familia del CIVB Infancia Feliz 


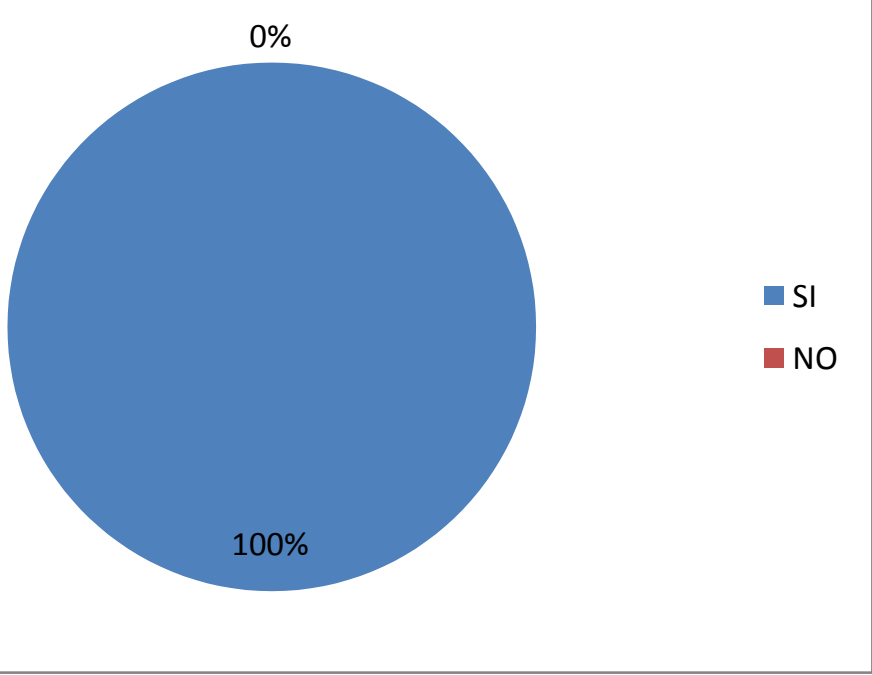

Grafico $n^{\circ}$ 9: la postura

Fuente: padres de familia del CIVB infancia feliz

\section{ANÁLISIS}

El $100 \%$ de los padres de familia mencionan que la postura que tiene su niño es correcta al realizar sus actividades cotidianas.

La postura corporal de los niños en las primeras etapas de la vida es crucial en su capacidad de adquirir nuevos conocimientos.

\begin{tabular}{|l|l|l|}
\hline INDICADOR & FRECUENCIA & PORCENTAJE \\
\hline SI & 25 & $100 \%$ \\
\hline NO & 0 & $0 \%$ \\
\hline TOTAL & 25 & $100 \%$ \\
\hline
\end{tabular}

10.-La estimulación en la motricidad gruesa fortalece los músculos y adquieren agilidad en los movimientos de su niño.

TABLA N.-10 ESTIMULACION EN LA MOTRICIDAD GRUESA (MUSCULOS Y

MOVIMIENTOS)

FUENTE: Padres de Familia del CIVB Infancia Feliz 


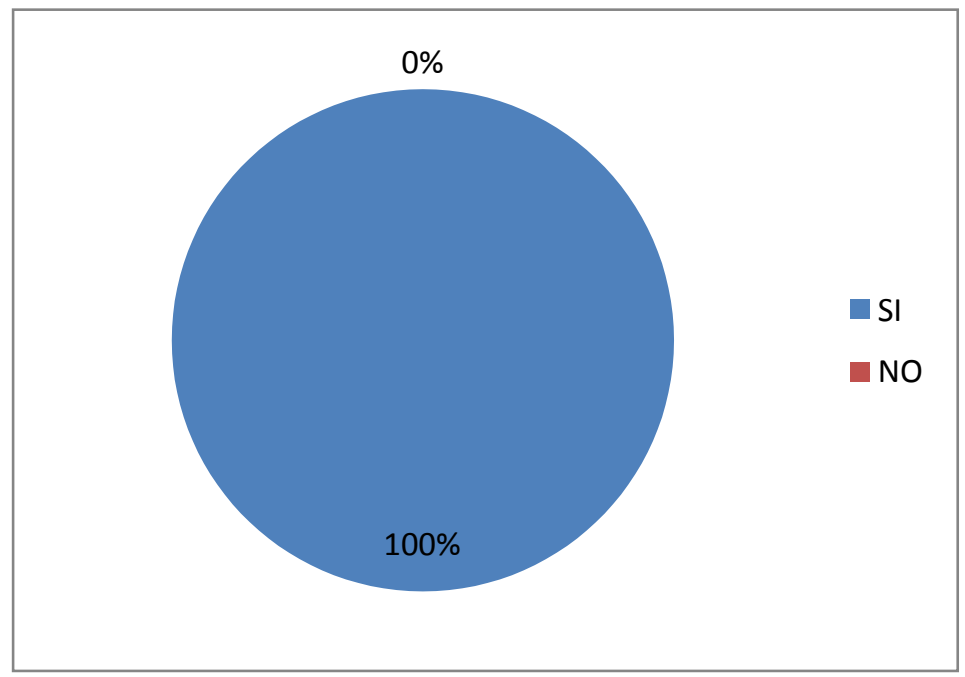

\section{Grafico n ${ }^{\circ}$ 10: estimulación en la motricidad gruesa (músculos y movimientos) Fuente: padres de familia del CIVB infancia feliz}

\section{ANÁLISIS}

El 100\% de los padres de familia del CIVB Infancia Feliz menciona que la estimulación en la motricidad gruesa fortalece los músculos y ayudan adquirir agilidad en los movimientos de su niño/a.

Al emplear una adecuada estimulación en motricidad gruesa vamos haciendo que el niño adquiriera una capacidad para mover armoniosamente los músculos de su cuerpo y mantener el equilibrio, además de adquirir agilidad, fuerza y velocidad en su movimiento.

Análisis e interpretación de la ficha de observación inicial aplicada a los niños y niñas de 24 a 36 meses del CIBV.

Se ha tomado 25 niños y niñas de 24 a 36 meses del CIBV para aplicar diez actividades propuestas en la ficha de observación la cual tiene como indicador de evaluación sí o no de los cuales detallaremos a continuación. 
El 36\% de los niños y niñas caminan sin dificultad, en línea recta, y en puntillas alternado los pies y el 64\% no logra realizar la actividad por lo cual no han desarrollado una adecuada estimulación en el área motriz gruesa.

La coordinación es un aspecto importante en esta etapa la cual ya debe ser estimulada para poder controlar la coordinación eso por eso que $44 \%$ de los niños/as suben las escaleras alternando los pies y en un 56\% aun no lo han logrado, para bajar las escaleras alternando los pies el $40 \%$ logra alternar los pies y el $60 \%$ no lo hace esto depende a que necesitaran una estimulación en el aspecto de coordinación mano-pie.

Al realizar la actividad de lanzar y recibir la pelota se pudo observar que el $32 \%$ logra realizar la actividad el $68 \%$ de los niños no logra hacer esta actividad ya que se le complica mucho al recibir la pelota.

El ejercicio de correr y saltar alternando los pies es complejo ya que necesita de coordinación, equilibrio y rapidez para lograrlo, de los niños/as que lo realizaron esta el 20\% y un $80 \%$ que aún no lo logran

El tener una coordinación y un equilibrio en los pies se torna un tanto difícil para los niños, y mucho más si se trata de caminar hacia atrás, por lo que un $48 \%$ de los niños si realizan esta actividad, mientras que el 52\% no puede caminar hacia atrás.

Dentro de una buena coordinación está el desarrollar ritmo en los movimientos que vaya adquiriendo el niño, por que el $44 \%$ de los infantes pueden realizar la actividad. Y el $56 \%$ no lo realiza ya que se le presenta dificultad en el momento de saltar con ritmo de un pie y de dos pies. 
María F. Constante-Barragan; Adriana G. Rengifo-Tapia; Leslie A. Herrera- Yugcha

El aspecto de coordinación es tomado en cuenta para proponer actividades ya que dan a notar si existe algún tipo de dificultad en la marcha coordinando pies y manos, el 24\% logran realizar la actividad propuesta y un $76 \%$ no realiza la actividad ya que su coordinación no está desarrollada completamente.

Para poder realizar actividades que presente diferentes dificultades como atravesar obstáculos, es importante a ver desarrollado una adecuada motricidad gruesa, el $40 \%$ de los niños pueden realizar esta actividad sin ningún tipo de dificultad, mientras que al $60 \%$ de los infantes se le torna difícil el atravesar obstáculos.

Realiza movimientos del cuerpo manteniendo equilibrio el 52\% de los niños lo pueden realizar sin ninguna dificultad ya que al empezar a caminar ya están manteniendo equilibrio corporal, y el $48 \%$ de los infantes aun no pueden controlar sus movimientos y mantener el equilibrio.

Análisis e interpretación de la ficha de observación final aplicada a los niños y niñas de 24 a 36 meses del CIBV.

Se ha tomado 25 niños y niñas de 24 a 36 meses del CIBV para aplicar diez actividades propuestas en la ficha de observación la cual tiene como indicador de evaluación sí o no de los cuales detallaremos a continuación.

El 68\% de los niños y niñas caminan sin dificultad, en línea recta, y en puntillas alternado los pies y el $32 \%$ no logra realizar la actividad por lo cual no han desarrollado una adecuada estimulación en el área motriz gruesa. 
La coordinación es un aspecto importante en esta etapa la cual ya debe ser controlada es por eso que $76 \%$ de los niños/as suben las escaleras alternando los pies y en un $24 \%$ aun no lo han logrado es por esto que la bajar las escaleras alternando los pies nos muestran el mismo porcentaje del $76 \%$ que logra alternar los pies y el $24 \%$ que no lo hace esto depende a que necesitaran una estimulación en el aspecto de coordinación mano-pie.

Al realizar la actividad de lanzar y recibir la pelota se pudo observar que el $88 \%$ ya logra realizar la actividad con un poco de dificultad pero un rango del $12 \%$ que es muy bajo aun no lo realiza.

El ejercicio de correr y saltar alternando los pies es complejo ya que necesita de coordinación, equilibrio y rapidez para lograrlo, de los niños/as que lo realizaron esta el 76\% y un $24 \%$ que aún no lo logra es por esto que la actividad de saltar varias veces con ritmo de un pie y dos pies nos da los mismos porcentajes ya que se necesitan los mismos elementos anteriores y nos da que el $76 \%$ lo hace y el $24 \%$ no lo hace lo que se pudo observar es que necesitan más estimulación en los elementos que constituyen la psicomotricidad.

El aspecto de coordinación es tomado en cuenta para proponer las actividades ya que se ven varias falencias como marcha coordinando pies y manos y atraviesa obstáculos sin inconvenientes nos dan resultados similares a lo anterior que el $76 \%$ que es un porcentaje grande ya logra las dos actividades propuestas y en un porcentaje menor del $24 \%$ no realiza la actividad lo que pondrán más interés en realizar estimulación adecuada.

Realizando el ejercicio de caminar hacia atrás que es complejo el $68 \%$ ya lo puede lograr sin inconvenientes y el $32 \%$ tiene dificultad ala realizar el ejercicio ya que el caminar de espalda no tienen seguridad 
María F. Constante-Barragan; Adriana G. Rengifo-Tapia; Leslie A. Herrera- Yugcha

Realiza movimientos del cuerpo manteniendo equilibrio el $100 \%$ de los niños lo pueden realizar sin ninguna dificultad ya que al empezar a caminar ya están manteniendo equilibrio corporal es por esto que en esta actividad no hubo inconvenientes para realizarlo.

\begin{tabular}{|c|c|c|c|c|}
\hline \multirow{3}{*}{$\begin{array}{l}\text { ACTIVIDADES } \\
\text { 1. Camina sin dificultad, en línea recta y en } \\
\text { puntillas alternado los pies }\end{array}$} & \multicolumn{2}{|c|}{ 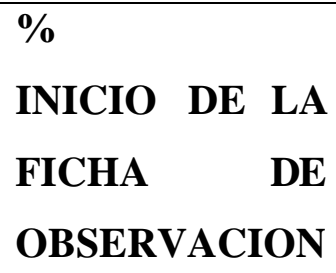 } & \multicolumn{2}{|c|}{$\begin{array}{lr}\% & \\
\text { FINAL DE } & \text { LA } \\
\text { FICHA } & \text { DE } \\
\text { OBSERVACION }\end{array}$} \\
\hline & SI & NO & SI & NO \\
\hline & $36 \%$ & $64 \%$ & $68 \%$ & $32 \%$ \\
\hline 2.Sube las escaleras alternado los pies & $44 \%$ & $56 \%$ & $76 \%$ & $24 \%$ \\
\hline $\begin{array}{l}\text { 3.Salta varias veces con ritmo de un pie y } \\
\text { dos pies }\end{array}$ & $40 \%$ & $60 \%$ & $76 \%$ & $24 \%$ \\
\hline 4.Lanza y recibe la pelota & $32 \%$ & $68 \%$ & $88 \%$ & $12 \%$ \\
\hline 5.Corre y salta alternando los pies & $20 \%$ & $80 \%$ & $76 \%$ & $24 \%$ \\
\hline 6. Camina hacia atrás & $48 \%$ & $52 \%$ & $76 \%$ & $24 \%$ \\
\hline 7. Sube las escaleras alternando los pies & $44 \%$ & $56 \%$ & $76 \%$ & $24 \%$ \\
\hline 8. Marcha coordinando pies y manos & $24 \%$ & $76 \%$ & $68 \%$ & $32 \%$ \\
\hline 9. Atraviesa obstáculos sin inconvenientes & $40 \%$ & $60 \%$ & $80 \%$ & $20 \%$ \\
\hline $\begin{array}{l}\text { 10. Realiza movimientos del cuerpo } \\
\text { manteniendo el equilibrio }\end{array}$ & $52 \%$ & $48 \%$ & $100 \%$ & $0 \%$ \\
\hline
\end{tabular}

Tabla 11 comparativa de la ficha de observación inicial y la ficha de observación final

\section{IMPACTOS (TECNICOS, SOCIALES, AMBIENTALES O ECONÓMICOS)}

El impacto que tiene el presente proyecto de investigación es social ya que busca un cambio en el desarrollo motriz de los niños/as de un centro que pertenecen a un colectivo social cuyos beneficios y a su vez obtener un progreso, a través de investigaciones realizadas, se verificar 
también como un cambio pueden ser el resultado de un proceso que en gran medida debe verse relacionado con la o las personas que lo realizan.

Debido a que en el CIVB "Infancia Feliz" a través de técnicas de fichas de observación, entrevistas y encuestas se determina que no existe un óptimo desarrollo en el área motriz gruesa por lo que se busca dar soluciones a esta problemática social en este caso desarrollar habilidades y destrezas motoras gruesa en los niños y niñas de educación inicial.

Por lo que como solución a este problema se requiere dar importancia a la estimulación motriz gruesa en los niños de 24 a 36 meses, se busca mejorar la motricidad gruesa para que el infante pueda desarrollar de una adecuada manera sus habilidades y destrezas.

\section{Conclusiones.}

No existe un conocimiento teórico o de experiencias de motricidad gruesa de los padres de familia es por ello que no ayuda al niño en un adecuado desarrollo en el hogar, lo que se evidencia en el movimiento, coordinación, equilibrio, postura de su cuerpo el momento del trabajo en el CIBV.

Al ingreso al CIBV los niños son evaluados el desarrollo motriz por la docente encarga pero la cual a posterior no realiza ningún seguimiento en este aspecto, ni plantea ejercicios dirigidos al área motriz gruesa ya que el centro no cuenta con un determinado rincón para esta área y los materiales son inadecuados para trabajar es por eso que se tiene que adaptar al medio que ofrece.

Al observar a los niños y niñas se puede verificar que no existe una actividad motora adecuada, por lo que no se realiza estimulación que beneficie al desarrollo corporal de los infantes. 


\section{Bibliografía.}

Black, M., Baqui, A., Zaman, K., McNary, S., \& Arifeen, S. (2007). Depressive symptoms among rural Bangladeshi mothers: implications for infant development. J Child Psychol Psychiatry, 48(1), 764772.

Cerdas Núñez, J., Polanco Hernández, A., \& Rojas Núñez, P. (2002). El niño entre cuatro y cinco años: características de su desarrollo socioemocional, psicomotriz y cognitivo-lingüístico. Educación, 169182.

Lev Vygotsky, L. (1985). Pensamiento y Lenguaje. Buenos Aires: Pléyade.

Osorio, E., Torres-Sánchez, L., Hernández, M., López-Carrillo, L., \& Schnaas, L. (2010). Estimulación en el hogar y desarrollo motor en niños mexicanos de 36 meses. Salud Pública de México, 52(1), 14-22.

Pic, V. (1989). El desarrollo mental del niño, en: Seis estudios de psicología. Barcelona: Ariel.

Schilder, E. (1982). Desarrollo perceptual y motor en los niños. Barcelona: Ediciones Paidos. 